\title{
Suppression of character in English story-reading: The case of Chinese students
}

\author{
$\mathrm{Na}$ Zhao \\ School of Foreign Languages, Kunming University, China, 650214
}

Key words: suppression of character, English story-reading, comprehension, the threshold effect, the EFL learners.

\begin{abstract}
The study, from the perspective of Structure Building Framework by Gensbacher, provides the basis interpretation of story-reading comprehension processing for L2 learners. The findings of two experiments, conducted with the aid of E-Prime Programming, revealed that (1) learners activate suppression mechanism to escape from the irrelevant information for tracking down the character in comprehending the cause-effect clue; (2) learners' language competence is provided with the interactive influence with the efficiency of suppression; and (3) the "threshold effect" appears in suppression of character due to the learners' language efficiency level.
\end{abstract}

\section{Introduction}

Reading is the avenue for acquiring knowledge and learning the world. Reading, for EFL (English as a lingua franca) learners, exhibits its importance in development of their language acquisition. Most, surrounded with an environment lacking in language-input, solely rely on reading for acquiring input (Zhang 2000). Therefore, English reading is gaining much attention from Linguistics and psycholinguistics. Story-reading, though, as a usual pattern for EFL testing, has rarely been laid a finger on both in abroad and domestic researches. How is the process of story-comprehension composed for EFL learners and how the learners construct the mental representations in reading which contains reservoirs of information, varied characters and complex plots (Kintsch 1998) are the focuses in the study. The investigation aims at helping improve L2 learners' reading comprehension efficiency by going into the suppression mechanic in story-reading based upon the Structure Building Framework proposed by Gernsbacher (1990).

\section{Related Literature}

\subsection{The Structure Building Framework}

Reading comprehension, based on SBF, is a generalized and consistent process of mental representations which construct the content of subject. SBF comprises three segmentations, namely, laying a foundation, mapping and shifting. In addition, the reader, first of all, constructs an elementary mental structure with the initial input; the subsequent information, afterwards, can be mapped on the meta-structure when they are correspondent with each other, but if they are at odds, the subsequent information is shifted into a sub-structure.

Furthermore, the memory cells, as the chunk of readers' mental representations, is activated by three elements, namely, (1) the input information; (2) the background information of the reader; (3) the linguistry of the reader (Walter 2007). Once the memory cells are activated, the information the memories carry is available in cognitive processing to convey signals that either suppress or intensify its memory cells. The extent of activating memory cells, as the result, is dependent on the suppression and the intensification functioning as the mediation.

The suppression and the intensification are crucial in the language processing. In the most cases, the irrelevant information is automatically activated or extracted unconsciously (Gernsbacher 1997). The reader thus should suppress the irrelevant information so as to comprehend without distortion. The series of researches concerning vocabulary, syntax and reading comprehension, conducted by Gernsbacher and other experts, indicate that the suppression is of great help for shielding the irrelevant information (Gernsbacher \& Shlesinger 1997; Gernsbacher et al, 20014). Therefore, the 
suppression for the readers, as the core of the Structure Building Framework, is bound to the differential comprehension (Gernsbacher 1993).

\subsection{The essential of story-reading comprehension}

The causal path, as the causal connection among events, is decisive in story reading. In the process of story-comprehending, the reader constructs a situational model (Radvansky et al. 2001), including roles, characters, mental state, actions for achieving goals, obstacles, conflicts, times and places (Graesser et al. 2002). The explicit and implied information should be inferred from the readers' background knowledge so clarifying the causal path hence is the essential to establish a plentiful and detailed situational model for comprehending stories smoothly and correctly.

The essentiality of the causal path has been authenticated by the fact that the information or the proposition relevant to causal connections is liable to being memorized, paid much attention by the reader, easily understandable and sustainable in short-term memory (Omanson 1982; Trabasso \& van den Broek 1985; Fletcher et al. 1990). If the complicated causal path, furthermore, is altered into a legible way to be followed, the reader presents high efficiency in reading comprehension (Linderholm et al. 2000). The informed research indicates that one of solutions to the information processing is to get characters' goals tracked down consistently (Linderholm et al. 2004; Virtue \& Czarlinske 2010).In story-reading, characters generally cling to their own goals which are the center for the causal path of events and for roles to conduct varied actions and lead to a variety of results.

To sum up above, comprehension is a process generalized and the Structure Building Framework is at work as a predictor in story-reading comprehension. Besides, how are the variables involved in story-reading comprehension and how is the process of comprehension at work must be concerned. The previous studies propose the "threshold effect", which expounds if learners' language competence level with the threshold, the reading skills used in reading stories written in mother language can be transferred. If not, the "short-circuit" phenomenon appears due to the influences of language competence and cognitive mechanisms, like suppression (Wu Shiyu \& Wang Tongshui 2006). The purpose of this study is to determine whether the effect reappears in story reading comprehension. Based upon this assumption, the present study investigates, by a series of tests, Chinese learners' concrete manifestations of constructing mental representation in suppression of English story-reading. The three research questions are the following:

(1) In story reading, whether learners intensify the initial character when repeatedly mentioned and whether they compress initial character, when new one introduced, to get the cause-effect clue?

(2) In process of comprehension, how learners' suppression and competence interact with each other?

(3) In story reading, whether the "threshold effect" appears when learners trigger out comprehension mechanism?

\section{Design}

Based upon the findings related to each question, two dependent variables tested in experiments are: (1) the reaction time the learners use in comprehension; (2) the error rate the learners make in judging target detective words. Therefore, the first experiment adopts the repeated ways of tests in 2 groups to measure the reaction time; the second utilizes tests among 3 groups to measure the reaction time and error rate.

\subsection{Subjects}

125 non-English major students (76 female, 49 male) were the participants, who were sophomores in colleges, recruited for the first experiment.

60 non-English major students ( 35 female, 25 male) were recruited to participate the second experiment, and were divided into 3 groups averagely and randomly. The results of English Competence Test for three groups indicated no marked difference. 


\subsection{Materials}

\subsubsection{English competence test}

The test was composed of vocabulary, grammar, reading comprehension and listening comprehension from CET 4 and CET 6 . The full score was 100 points.

\subsubsection{Suppression in story-reading test}

The test was accomplished with 10 syllogistic passages with 4 sentences and 130-150 words for each. The passage consisted of 3 paragraphs. The first was introduction paragraph in which the role was introduced in the first sentence; the second were presented in 3 ways, namely the initial role repeatedly mentioned in the story, introduction to new role and the neutral information (neither repeating the initial role nor introducing the new role), and the third was conclusion. The first and the third were the same for all students.

\subsubsection{Story reading test}

Each passage was attached with 5 statements for judging "True" or "False" and 1 question for choosing correct one from multiple choices. The score was 1 point for each statement and 2 points for the question separately.

Two experiments were conducted with the same measurement and by the same procedures. Moreover, the second consisted of 3 sets of passages according to the arrangement of writing, and each group of students read one of them.

\subsection{Data collection}

3.3.1 English competence test was taken firstly and time length was 120 minutes.

3.3.2 Suppression test was taken in the language laboratory and each student finished reading passages complied with E-Prime programming beforehand. Stories were presented on the screen one paragraph after another and at each interval between two paragraphs, the detective words appeared, then students determined whether they had read before or not. In this case, the information of characters in their memories could be accessible. Students pressed "1" for "yes" and " 2 " for "no" to measure the reaction time recorded on the computer simultaneously.

\subsubsection{Story reading test was taken on computer and scores were accessible.}

The latter experiments were to be accomplished in the lab, the time length was 50 minutes. After that, all datum was imported into SPSS to have treatment of the data.

\section{Findings}

\section{1 the first experiments}

Table 1 revealed that the descriptive statistics about the scores students had achieved in English language competence test and story-reading test, including two groups with different language proficiency levels. The scores for story-reading test was the total points for 3 passages.

Table 1: the descriptive statistics of the English competence test and story-reading test

\begin{tabular}{lcccccc}
\hline & N & Max & Min & Mean & SD & sig \\
\hline English competence test & & & & & & \\
Total & 125 & 90 & 23 & 61.21 & 11.26 & .82 \\
Group with higher efficiency level & 52 & 90 & 65 & 72.02 & 3.82 & \\
Group with lower efficiency level & 43 & 59 & 23 & 42.83 & 6.64 & \\
\hline Story-reading test & & & & & & \\
Total & 125 & 21 & 6 & 14.70 & 3.45 & .72 \\
Group with higher efficiency level & 52 & 21 & 6 & 15.33 & 3.07 & \\
Group with lower efficiency level & 43 & 18 & 6 & 11.88 & 3.82 & \\
\hline
\end{tabular}


Table 2 covered the mean and the SD concerning the reaction time students used in judging target detective words. Furthermore, datum in table 2 were tested by ANCOVA (Analysis of Covariance) to check the existence of main effect. The target character, as the independent variable, comprised of 3 levels, namely, the initial character repeatedly mentioned, the new character and the neutral information while the reaction time in judging detective words was the dependent variable. The language competence and the score of reading comprehension test, as a covariant variable, were brought into the analytic model. However, the competence and the score presented high degree of association $(r=.84, \mathrm{p}=.000)$, so the score was excluded from the model.

Table 2: the descriptive statistics of the reaction time in judging detective words

conditions the reaction time in judging detective words (MS)

\begin{tabular}{lcccc} 
& Max & Min & Mean & SD \\
\cline { 2 - 5 } & & & & \\
Total (125) & 3407.50 & 983.20 & 1783.24 & 613.65 \\
The initial target firstly mentioned & 2093.20 & 983.20 & 1638.35 & 392.01 \\
The initial target repeatedly mentioned & 3308.25 & 1089.05 & 2988.90 & 545.83 \\
The new target & 3365.08 & 1206.54 & 1639.75 & 509.26 \\
Neutral information & & & & \\
& & & & \\
\hline Group with higher efficiency level (52) & 2257.08 & 983.20 & 1742.09 & 715.62 \\
The initial target firstly mentioned & 2198.46 & 916.53 & 2638.62 & 789.02 \\
The initial target repeatedly mentioned & 2480.52 & 1152.35 & 1960.88 & 690.35 \\
The new target & 2965.37 & 869.40 & & \\
Neutral information & & & 3019.34 & 620.46 \\
& & & 2053.74 & 703.26 \\
Group with lower efficiency level (43) & 3452.50 & 2245.80 & 2809.14 & 696.58 \\
The initial target firstly mentioned & 3024.90 & 1935.30 & 2374.58 & 652.05 \\
The initial target repeatedly mentioned & 3537.00 & 2245.20 & &
\end{tabular}

Meanwhile, the data collected in the repeated tests, were tested to check the condition to meet the Huynh Feldt KMO (Kaiser-Meyer-Olkin) test. The result, though, was $p=.001$, so the study adopted Huynh-Feldt Epsilon to correct the degree of freedom. The findings revealed the target character was provided with the main effect $(\mathrm{F}(1.996,320.226)=26.99, \mathrm{p}=.000)$. Accordingly, the null hypothesis regarding the influence on the target role from variables was invalid.

It was found that students had evidently spent longer reaction time when the new character was introduced to the story, which was indicated in each group and among 3 groups as well. In the development of stories, students had spent less reaction time to the initial character than to the new character and the neutral information $(\mathrm{F}(1,048)=46.52, \mathrm{p}=.000 ; \mathrm{F}(1,048)=18.53, \mathrm{p}=.000)$, which made it clear that the initial target character was intensified increasingly in the mental representation structure when it was mentioned continuously. Subsequently, the students were able to extract the information relevant to the target role more easily and spent less time. Besides, the students spent more time responding to the new character than to the neutral information $(\mathrm{F}(1,048)$ $=9.84, \mathrm{p}=.000)$. The students, under this circumstance, suppressed the previous information to track down the clue of new character. Subsequently, the students had to weaken the intervention of the initial character and then spent more time reacting to the new information.

The findings, furthermore, suggested that students had spent longer time responding to the initial character at the first time than to the initial character mentioned later $(\mathrm{F}(1,048)=2.92, \mathrm{p}=.005)$, which clarified that the language competence, as a significant variable, was to be under consideration in foreign language reading.

The study, on the basis of the findings, suggested that EFL learners had tracked down the target character that repeatedly mentioned and suppressed the previous and irrelevant information so as to maintain the track down to the cause-effect clue for comprehending stories.

The study also supported the idea that the learners' suppression had marked interaction effect with their language competence $(\mathrm{F}(1,863,310.473)=3.16, \mathrm{p}=.03)$. The conclusion, from another perspective, was authenticated by measuring the time difference between the reaction time of responding to the new role and to the neutral information, which is regarded as measurement of the 
suppression. The higher learners' proficiency level is, the more capable they are to suppress the irrelevant information for bettering comprehension.

To the third hypothesis, students were divided into two groups, namely, group 1 with higher proficiency level and group 2 with lower level as shown in the table 1 . The analyses of the tests in three different conditions revealed that the suppression possesses the main effect $(F(2,025)=11.06$, $\mathrm{p}=.000$ ) and the data collected in the experiments for students with lower efficiency level suggested that suppression fail to possess the main effect $(\mathrm{F}(1.63,87.25)=.81, \mathrm{p}=.53)$, which disclosed that the group with higher efficiency level were more capable to suppress the irrelevant information. The conclusion was the same as the "threshold effect" that illuminated the threshold of language, which influenced the activation of compression in comprehension.

\section{2 the second experiments}

60 students, divided into 3 groups, participated in testing reaction time and the error rate in judging detective words. The findings, tested by one-way ANOVA, suggested that the marked difference among 3 group existed $(\mathrm{F}(2.76)=11.08, \mathrm{p}=.000)$. In addition, the findings indicated that $(1)$ the error rate was lower when the initial character was mentioned repeatedly than when the new character was introduced $(\mathrm{p}=.000)$ and when the neutral information appeared $(\mathrm{p}=.004)$; and (2) when the new character was introduced, the error rate was higher than not only the initial role but the neutral information. Students, in the case, were to suppress the initial character to track down the clue but it is more likely to make errors.

Table 3: the descriptive statistics of reaction time and error rate the mean of reaction time (SD) the mean of error rate (SD)

\begin{tabular}{llccc}
\hline & pre-suppression & post-suppression & pre-suppression & post-suppression \\
\hline condition 1 & $1755.58(698.27)$ & $2294.80(778.25)$ & $20.78(7.06)$ & $26.98(14.65)$ \\
condition 2 & $1833.05(526.53)$ & $3567.22(706.93)$ & $22.86(6.86)$ & $47.20(14.09)$ \\
condition 3 & $1738.92(683.80)$ & $3116.85(560.30)$ & $19.92(6.90)$ & $38.74(12.49)$
\end{tabular}

P.S.: condition $1=$ the initial target repeatedly mentioned; condition $2=$ the new target; condition $3=$ the neutral information

The second experiment furthered to approve the findings of the first one, namely the function of suppression in story reading.

\section{Discussion}

The Structure Building Framework interprets how the readers construct the basic mental representation by utilizing the character mentioned initially in stories and how the readers subsequently reflect the information relevant to the mental representation onto the structure. Accordingly, the mental representation is intensified if the initial target is repeatedly mentioned; when, however, the new target is introduced, readers transfer their attention to build up a new sub-structure by restraining the existing comprehension and escaping from the interference of irrelevant information. The findings of the study indicate the evident relations between intensification and suppression. The "profit earning" can be reaped when the initial target is mentioned repeatedly. In this case, readers make quicker response to the target and score lower error rate. On the contrary, the "cost" should be paid when the new target is introduced in the reading. In this case, readers make slower response to the target and score higher error rate. Thus, to comprehend the story efficiently is bound to the advantages of repeated information and the reduction of interference from the irrelevant information.

The result of the study is the presentation of the landscape model of reading by Linderholm (2004). The landscape model of reading is the connectionist theory, a landscape of dynamic transfer and activation, referring to the process of activating concepts that presents changes in volatility. The process is influenced by two primary elements, namely, the learners' attention limit and the effort the learners make to maintain a coherent representation. The learners, thus, need to activate their working memory to maintain information they have read. For best understanding, moreover, learners have to acquire the information relevant to the cause-effect clue in order to activate 
information kept in memory rather than to suppress the irrelevant information to release some memory room for more information. The purpose of the study, from multiple perspectives, is to indicate the process of suppression representation in stories reading. The initial character, as the relevant information to the cause-effect clue, is to be intensified and activated repeatedly in the memory representation when it repeatedly mentioned; when the new character is introduced to the story, the initial character is to be compressed due to its irrelevance to latter comprehension. In the case, more attention is paid to the new target so as to acquire better comprehension.

In addition, the findings of the study make it clear that the learners' language competence is crucial in processing reading. The higher the learners' competence level is, the more capable the learners are in suppressing the irrelevant information and comprehending stories. Apart from mother language acquisition, foreign language acquisition is interrelated to the learners' language competence, which has direct or indirect impact on reading comprehension.

\section{Conclusion}

The findings of the study provide the basis that story reading is a generalized process, involved in the readers' coherent representation in reading and support the idea that the target character is crucial in comprehending stories. Readers, relying on the suppression mechanism, track down the relevant information to obtain the cause-effect clue for comprehending stories. In the process, the suppression mechanism functions as a checker to ensure the relevant information kept in the working memory and prevent the irrelevant information from maintaining in the working memory (Wu Shiyu 2009). The study also suggests that readers maintain the target activated before the new target appears. Once the new target appears, readers weaken the extent of activation. Therefore, the suppression mechanism in reading takes charge in constructing the readers' comprehension representation.

\section{References:}

[1] Fletcher, C., J. Hummel \& C. Marsolek. 1990. Causality and the allocation of attention during comprehension [J]. Journal of Experimental Psychology 16:233-240;

[2] Gernsbacher, M. 1990. Language Comprehension as Structure Building[M]. Hillsdale, N.J.: Erlbaum.

[3] Gernsbacher, M. 1993. Less skilled readers have less efficient suppression mechanisms [J]. Psychological Science 4: 294-298.

[4] Gernsbacher, M. 1997. Two decades of structure building [J]. Discourse Processes 23: 265-304.

[5] Gernsbacher, M. \& M. Shlesinger. 1997. The proposed role of suppression in simultaneous interpretation [J]. Interpreting 2: 119-140.

[6] Graesser, A., B. Olde \& B. Klettke. 2002, How does the mind construct and represent stories [A]. In G. Melanie, J. Strange \& T. Brock (eds.). Narrative Impact $[\mathrm{C}]$. Mahwah, N. J.: Erlbaum. 1-51.

[7] Linderholm, T. et al. 2004. Suppression of story character goals during comprehension [J]. Discourse Processes 37: 67-78.

[8] McNamara, D. \& M. McDaniel. 2004. Suppressing irrelevant information: Knowledge activation or inhibition? [J]. Journal of Experimental Psychology 30: 326-337.

[9] Walter, C. 2004. Transfer of reading comprehension skills to L2 is linked to mental representations of text and to L2 working memory [J]. Applied Linguistics 25: 315-339.

[10] Zhang, L. 2000. Metacognition in L2 reading literacy acquisition: The case of ten Chinses tertiary students learning to read EFL [A]. In A. Brown (ed.), English in Southeast Asia'99: 
Developing Multiliteracies[C]. Singapore: Nanyang Technological University. 83-96.

[11] Wu Shiyu. \& Wang Tongshun. 2006. The Research on the Transfer of Reading Skills in Foreign Language Reading from the Perspective of the Structure Building Framework.[J]. Foreign Languages Teaching and Research 2: 122-128. 